Originally published as:

Fabian, H., Huser, H., Loll, B., Ziegler, A., Naumann, D. and Uchanska-Ziegler, B. (2010), HLAB27 heavy chains distinguished by a micropolymorphism exhibit differential flexibility. Arthritis \& Rheumatism, 62: 978-987.

DOI: 10.1002/art.27316

This is an author manuscript.

The definitive version is available at: http://onlinelibrary.wiley.com 


\title{
HLA-B27 Heavy Chains Distinguished by a Micropolymorphism Exhibit Differential Flexibility
}

\author{
Heinz Fabian, ${ }^{1}$ Hans Huser, ${ }^{2}$ Bernhard Loll, ${ }^{3}$ Andreas Ziegler, ${ }^{2}$ Dieter Naumann, ${ }^{1}$ \\ and Barbara Uchanska-Ziegler ${ }^{2}$
}

Supported by the DFG (grants Na226/12-3, UC8/1-2, and SFB 449 B6), the Volkswagen Foundation (grant I/79 989), and the Robert Koch Institute.

${ }_{2}^{1}$ Robert Koch Institute, Berlin, Germany
${ }^{2}$ Charité-Universitätsmedizin Berlin and Freie Universität Berlin, Berlin, Germany
${ }^{3}$ Max Planck Institute for Medical Research, Heidelberg, Germany.

Address correspondence and reprint requests to Heinz Fabian, PhD, Robert Koch Institut, P 25, Nordufer 20, 13353 Berlin, Germany (e-mail: fabianh@rki.de); or to Andreas Ziegler, PhD, Institut für Immungenetik, Charité-Universitätsmedizin Berlin, Freie Universität Berlin, Thielallee 73, 14195 Berlin, Germany (e-mail: andreas.ziegler@charite.de).

Submitted for publication September 7, 2009; accepted in revised form December 9, 2009.

\begin{abstract}
Objective: Although the products of the HLA subtypes $B^{\star} 2705$ and $B^{\star} 2709$ differ only in residue 116 (Asp versus His) within their peptide-binding grooves, they are differentially associated with inflammatory rheumatic diseases such as ankylosing spondylitis (AS): B*2705 occurs in AS patients, whereas $B \star 2709$ is only rarely encountered. The reasons for this distinct association are still unclear but could include subtypespecific conformational and dynamic properties of these antigens. The present study was undertaken to investigate structural and dynamic differences between $B * 2705$ and $B \star 2709$ and their possible relationship to subtypespecific disease association.

Methods: The membrane-distal segments of the $B^{\star} 2705$ and $B^{\star} 2709$ heavy chains were expressed in vitro and reconstituted together with $B_{2}$-microglobulin and a peptide. HLA-B27 complexes loaded with 2 self peptides (TIS [RRLPIFSRL] and pVIPR [RRKWRRWHL]) and a sequence-related viral peptide ( $p L M P 2$ [RRRWRRLTV]) were studied by isotope-edited infrared spectroscopy to detect differences in their structure and flexibility at physiologic temperature.

Results: Our analyses revealed the existence of subtype-specific conformational differences between the 2 HLA-B27 heavy chains at physiologic temperature, which are undetectable using x-ray crystallography. Irrespective of the bound peptide, the heavy chain of the $B^{\star} 2705$ complex exhibited higher conformational flexibility than the $B * 2709$ heavy chain.

Conclusion: The present study demonstrates the existence of previously undetected systematic conformational and dynamic differences between the heavy chains of the 2 HLA-B27 subtypes. Since effector cell recognition of cells expressing HLA antigens is dependent on the dynamic properties of the interacting cell surface molecules, this HLA-B27 subtype-specific heavy chain flexibility could have a role in the distinct association of HLA-B27 subtypes with spondylarthritides.
\end{abstract}

The HLA class I allele (subtype) HLA-B*2705 is strongly associated with ankylosing spondylitis (AS), an inflammatory rheumatic disorder, whereas another HLA-B27 allele, HLA-B*2709, exhibits at most a weak association (1-3). The 2 encoded proteins differ only by a micropolymorphism (Asp-116 in $B^{*} 2705$ and His-116 in $B^{*} 2709$ ) (4) within the peptide-binding groove that is formed by each of the heavy chains $(5,6)$. Pairwise comparison of the functional, biochemical, and biophysical properties of these very closely related subtypes may thus shed light on the mechanisms underlying the differential disease association, although a haplotypedependent influence of B27-linked genes also cannot be excluded at present (7).

The exchange of residue 116 within the HLA-B27 heavy chain has already been found to alter recognition of the subtypes by cytotoxic T lymphocytes $(8)$ and the repertoire of bound peptides $(9,10)$. The presentation mode of selected peptides by each of the 2 subtypes is affected as well, as shown by x-ray crystallography $(11-15)$. The crystal structures suggest that the differential reactivity of cytotoxic T lymphocytes with the 2 HLA-B27 subtypes might be due primarily to the presence of 
different conformations of the peptides. Studies of HLA-B27 misfolding (16), heavy chain dimerization (17), and HLA-B27/human $\beta_{2}$-microglobulin $\left(\beta_{2} \mathrm{~m}\right)$-transgenic rats (18) have focused exclusively on the $B^{*} 2705$ subtype, and it is not known whether comparable experiments with $B^{*} 2709$ would have yielded different results. Apart from the identification of a self peptide that binds only to AS-associated HLA-B27 subtypes and represents a candidate for molecular mimicry with a Chlamydia trachomatisderived peptide (19), a unifying feature that would distinguish $B^{*} 2705$ from

$B^{\star} 2709$ and provide an explanation for the association of HLA-B27 with spondylarthritides has not as yet been identified.

Although there may be considerable conformational differences between the peptides displayed, a comparison of the $x$-ray structures of the $B^{*} 2709$ and $B^{*} 2705$ subtypes reveals that the structures of their heavy chains, as well as those of the noncovalently associated, invariant light chain of the

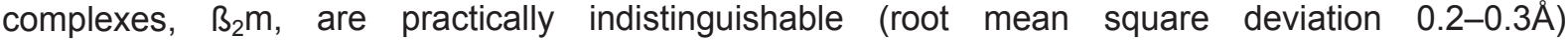
$(11,12,15,20)$. Likewise, spectroscopic analyses have not resolved whether distinct properties of

the subtypes' heavy chains could be generalized or were due only to the presence of a particular peptide within the binding groove $(15,21-24)$. Although these and other analyses provided more thorough understanding of many of the biophysical properties of HLA-B27 molecules, findings of structure-based studies have not been able to comprehensively account for the differential involvement of HLA-B27 subtypes in the pathogenesis of AS. Since experiments with transgenic rats revealed that the $B 27-\beta_{2} m$ complex can cause spondylarthritis $(18,25)$, further studies of the molecule's biophysical and functional properties are mandatory.

X-ray crystallography can provide only very limited information on protein dynamics, and since hydrogen atoms of these molecules (roughly $50 \%$ of all atoms) are virtually "invisible" due to insufficient resolution of the diffraction data, structural information should be supplemented using complementary approaches. Determination of specific absorption of infrared (IR) light by spectroscopy is a sensitive way to monitor conformational

changes within proteins or conformational differences between related proteins $(26,27)$. Data can be gained from $\mathrm{C}=\mathrm{O}$ stretching vibrations of amide carbonyl groups (amide I), $\mathrm{N}-\mathrm{H}$ bending vibration coupled to $\mathrm{C}-\mathrm{N}$ stretching (amide II), and $\mathrm{N}-\mathrm{H}$ stretching vibration of the peptide groups (amide $\mathrm{A}$ ). Moreover, the solvent accessibility of the protein backbone can be probed by hydrogen/deuterium $(\mathrm{H} / \mathrm{D})$ exchange experiments, thus providing additional information on the flexibility and dynamics of the molecule $(27,28)$. Therefore, the results obtained are distinct from the largely static picture obtained with conventional $x$-ray crystallography and, additionally, supply information on hydrogen atoms within a protein.

Using isotope-edited IR spectroscopic analyses with $13 \mathrm{C}$-labeled $\beta_{2} \mathrm{~m}$, unlabeled heavy chain, and 3 peptides, we demonstrated in the present study that the $B^{\star} 2705$ and $B^{\star} 2709$ subtypes exhibit previously undetected systematic differences in conformation and flexibility

at physiologic temperature. We discuss how differential heavy chain dynamics could influence the recognition of cells expressing HLA antigens by effector cells.

\section{Materials and Methods}

Sample preparation. The peptides TIS (RRLPIFSRL, derived from epidermal growth factor response factor 1), pLMP2 (RRRWRRLTV, derived from latent membrane protein 2 of Epstein-Barr virus), and pVIPR (RRKWRRWHL, derived from vasoactive intestinal peptide type 1 receptor) were synthesized and purified as described previously $(11,12,15)$. The extracellular domains of $B^{\star} 2705$ and $B^{\star} 2709$ as well as 13C-labeled $\beta_{2} m$ were expressed separately in Escherichia coli as inclusion bodies $(24,29)$. To obtain 13C-labeled $B_{2} m, E$ coli cells were cultured in minimal medium containing uniformly $13 \mathrm{C}$ labeled glucose (Cambridge Isotope Laboratories, Andover, MA) as described previously (24).

For complex formation, the proteins were solubilised with urea and refolded by dilution in the presence of the peptide $(29,30)$. Following reconstitution, the entire mixture was concentrated using Amicon Ultra-15 devices with a 10-kd cutoff (Millipore, Billerica, MA), and the complexes were isolated by sizeexclusion chromatography in $10 \mathrm{mM}$ sodium phosphate buffer $(\mathrm{pH} 7.5) / 150 \mathrm{mM} \mathrm{NaCl}$. For IR measurements, the sample solutions were concentrated and exchanged into the corresponding $\mathrm{D}_{2} \mathrm{O}$ buffer using Vivaspin 500 concentrators (Sartorius, Göttingen, Germany) with a membrane cutoff of 10 $\mathrm{kd}$. The final sample concentrations were between 10 and $20 \mathrm{mg} / \mathrm{ml}$ before collection of IR data. 
Infrared spectroscopy. The protein solutions were always freshly prepared and placed into demountable calcium fluoride IR cells with an optical path length of $50 \mu \mathrm{m}$ for measurements in $D_{2} \mathrm{O}$ buffer (26). IR spectra were recorded with an IFS-28B Fourier transform infrared spectrometer (Bruker Optics, Ettlingen, Germany) equipped with a DTGS detector and continuously purged with dry air. For each sample, 128 interferograms were co-added and Fouriertransformed to yield spectra with a nominal resolution of $4 \mathrm{~cm}^{-1}$. The sample temperature was controlled with a thermostated cell jacket. Spectra at discrete temperatures were obtained by heating the protein solutions from $15^{\circ} \mathrm{C}$ to $90^{\circ} \mathrm{C}$ in steps of $2.5^{\circ} \mathrm{C}$. Buffer spectra were recorded under identical conditions in a matched second cell of slightly reduced path length and subtracted from the spectra of the proteins in the relevant buffer. Spectral contributions from residual water vapor, if present, were eliminated using a set of water vapor spectra. The final unsmoothed protein spectra were used for further analysis. Band positions were determined using Opus software (Bruker). Second derivatives were obtained using the Savitzky-Golay algorithm with 13-point smoothing. The entire procedure has been described in detail previously (24).

\section{Results}

Choice of B27-peptide complexes. For the present studies, we selected the soluble, recombinant, membrane-extrinsic domains of the $B^{*} 2705$ and $B^{*} 2709$ subtypes complexed with 3 ligands. The ligands comprised the self peptides TIS $(15)$ and $\operatorname{PVIPR}(8,11)$, as well as the viral peptide pLMP2 (12). These peptides were chosen because high-resolution $\mathrm{x}$-ray crystallography had revealed that the TIS peptide is displayed very similarly by the 2 HLA-B27 subtypes, while the pLMP2 peptide and the pVIPR peptide exhibit drastically different conformations (Figure 1). In addition, pVIPR is bound by $B^{\star} 2709$ in a conventional single conformation, but by $B^{*} 2705$ in an exceptional dual conformation. These distinct modes of antigen presentation link the emergence of dissimilar, B27-specific $\mathrm{T}$ cell repertoires in individuals with the different subtypes to the buried Asp116His polymorphism $(8,11,12)$.

Infrared absorbance spectra of B27-TIS complexes. We initially investigated the IR spectroscopic behavior of the $B^{*} 2709-{ }^{13} C-B_{2} m-T I S$ and $B^{*} 2705-{ }^{13} C-B_{2} m-T I S$ complexes after transfer into $D_{2} O$ buffer (Figure 2), because only the TIS peptide is bound by both subtypes in a nearly identical conformation $(6,15)$ (Figure 1). The spectra were very similar to those previously determined for complexes with pVIPR (24), and revealed a feature at $\sim 1,594 \mathrm{~cm}^{-1}$ due to ${ }^{13} \mathrm{C}$-labeled $\beta_{2} \mathrm{~m}$ as well as several heavy chain-specific band components between 1,620 and $1,700 \mathrm{~cm}^{-1}$. The spectral attributes of the 9 residues of the TIS peptide were "buried" under those of the 276 heavy chain residues, indicating that practically all spectral changes observed must be regarded as contributions of the heavy chain. The remaining IR intensity at $\sim 1,545 \mathrm{~cm}^{-1} 1$ hour after transfer into $\mathrm{D}_{2} \mathrm{O}$ buffer, together with the presence of a band at $\sim 3,286 \mathrm{~cm}^{-1}$, showed that a number of amide $\mathrm{N}-\mathrm{H}$ groups of the heavy chain in the complex are protected from H/D exchange. The band at $\sim 3,286 \mathrm{~cm}^{-1}$ (amide

A) was the best indicator of residual nonexchanged $\mathrm{N}-\mathrm{H}$ groups, owing to the lack of other protein absorption in the range $3,200-3,400 \mathrm{~cm}^{-1}$.

Analogous studies were also carried out with complexes of each HLA-B27 subtype and pLMP2 (results not shown), in all cases revealing $\beta_{2} \mathrm{~m}$ - and heavy chain-specific spectral features similar or even identical to those observed with complexes with TIS (present

study) or pVIPR (24). Together with the second derivatives of the spectra (thin traces in Figure 2 provide examples for the 2 complexes with the TIS peptide), these spectral properties form the basis for the comparative analyses described below.

Detection of HLA-B27 subtype-dependent conformational properties. IR difference spectra $\left(B^{*} 2709-{ }^{13} \mathrm{C}-\beta_{2} \mathrm{~m}\right.$-peptide spectra minus $\mathrm{B}^{*} 2705-{ }^{13} \mathrm{C}-\beta_{2} \mathrm{~m}$ - peptide spectra) obtained at different temperatures were then used to detect the possible presence of subtype-specific conformational features (Figure 3). Positive and negative components in these spectra reflect fine structural differences, in whose absence only a flat line is obtained. At $15^{\circ} \mathrm{C}$, the spectral characteristics in the amide $A$ region (a positive feature at $\sim 3,286 \mathrm{~cm}^{-1}$ ) and in the amide II/II' region (a positive band near $1,550 \mathrm{~cm}^{-1}$ and a corresponding negative band near $1,445 \mathrm{~cm}^{-1}$ ) demonstrated the presence of fewer $\mathrm{H} /$ Dexchanged amide groups in the heavy chain of $\mathrm{B}^{*} 2709$ compared with the $\mathrm{B}^{*} 2705$ subtype. More specifically, a positive feature at $1,651 \mathrm{~cm}^{-1}$, along with a negative component at $\sim 1,640 \mathrm{~cm}^{-1}$, suggests the presence of more nonexchanged amide groups within the helical regions of the complex in $B^{*} 2709$ than in $B^{\star} 2705$. Since less $H / D$ exchange is an indicator of a diminished degree of protein flexibility and, in turn, a sign of reduced accessibility of (inner) regions of a polypeptide to solvent molecules, these IR data demonstrated that the $B^{\star} 2705$ heavy chain is more flexible than the $B^{\star} 2709$ 
heavy chain.

The weak difference features around 1,690 and $1,621 \mathrm{~cm}^{-1}$ indicated minor differences in peak position of the amide I' bands, attributable to the ß-sheets of the heavy chain. In contrast, only minor positive and negative features due to the $\beta$-sheet band of ${ }^{13} \mathrm{C}$-labeled $\beta_{2} \mathrm{~m}$ (near $1,590 \mathrm{~cm}^{-1}$ ) were observed, indicating a very similar degree of $H / D$ exchange of the amide protons of $\beta_{2} m$ in complex with $\mathrm{B}^{\star} 2705$ or $\mathrm{B}^{\star} 2709$.

The IR difference spectroscopic features did not change appreciably between $15^{\circ} \mathrm{C}$ and $55^{\circ} \mathrm{C}$ (Figure 3 ). In contrast, very pronounced differences were observed at $60-65^{\circ} \mathrm{C}$ (Figure 3). The positive features of the IR difference spectrum at these elevated temperatures can be attributed to helical $\left(1,640 \mathrm{~cm}^{-1}\right)$ and antiparallel B-sheet $\left(1,688\right.$ and $\left.1,621 \mathrm{~cm}^{-1}\right)$ heavy chain structures (24), which indicate a higher amount of residual secondary structure in the $B^{\star} 2709$ complex with the TIS peptide than in the $B^{*} 2705$ complex with the TIS peptide. This distinguishing characteristic is a consequence of the higher thermostability of the $B^{*} 2709-T I S$ complex (15). The secondary structure was, in both cases, disrupted upon further temperature increases to $>70^{\circ} \mathrm{C}$, leveling out all spectral differences (lower trace in Figure 3). IR difference spectra were also obtained

from the complexes of both HLA-B27 subtypes with pLMP2 and pVIPR (results not shown), revealing the presence of heavy chain-specific spectral features as well, that were characteristic for a given HLA-B27 subtype. Taken together these data demonstrated that the observed spectral differences are independent from the sequence or conformation of the TIS, pLMP2, or pVIPR peptides in a given complex.

Discrete dissimilarities between the spectral characteristics of the HLA-B27 subtypes. The quality of the original IR absorbance spectra allowed us to compute differences between the second derivative spectra. This enabled us to visualize fine differences in the position, intensity, and shape of band components in more detail than would be obtained by simple comparison of the original spectra.

The low-temperature IR difference spectrum obtained by subtracting the second derivative IR spectrum of $B^{*} 2705-{ }^{13} C-B_{2} m-$ TIS from that of $B^{*} 2709-{ }^{13} C-B_{2} m-T I S$ (Figure $4 A$ ) revealed a number of positive and negative features between $1,700 \mathrm{~cm}^{-1}$ and $1,610 \mathrm{~cm}^{-1}$, reflecting characteristic structural differences between the heavy chains of the 2 HLA-B27 subtypes. Specifically, the negative feature at $1,650 \mathrm{~cm}^{-1}$ revealed a more intense and/or sharper amide I band component in the spectrum of $B^{*} 2709$ compared with that of $B^{*} 2705$, corroborating the conclusions derived from analyses of the original spectra (Figure 3), i.e., that there are more nonexchanged amide groups present in the helical regions of the $B^{\star} 2709$ than the $B^{\star} 2705$ complexes. Differences in peak position of the high-frequency B-sheet band components at $1,691-1,693 \mathrm{~cm}^{-1}$, together with the minor spectral differences of the lowfrequency $ß$-sheet band at $\sim 1,623 \mathrm{~cm}^{-1}$, indicate the presence of fine

differences in hydrogen-bonding patterns of the $B$-sheet structures of the heavy chain in the $2 \mathrm{HLA}-$ B27 samples at low temperatures. In marked contrast, the hightemperature IR difference spectrum was featureless (blue trace in Figure $4 \mathrm{~A}$ ) due to a loss of all conformational and dynamic differences between the 2 HLA-B27 heavy chain subtypes at $90^{\circ} \mathrm{C}$.

We next investigated whether conformational

differences between the heavy chains of the $B^{*} 2709$ and $B^{*} 2705$ subtypes could also be found in complexes with the pLMP2 peptide. Unlike the TIS peptide, this virusderived ligand is bound by the 2 HLA-B27 subtypes in very dissimilar conformations (12) (Figure 1 ). However, the spectra of $B^{*} 2709$ ${ }^{13} \mathrm{C}-\beta_{2} \mathrm{~m}-\mathrm{pLMP} 2$ and $\mathrm{B}^{*} 2705-{ }^{13} \mathrm{C}-\mathrm{B}_{2} \mathrm{~m}-\mathrm{pLMP} 2$ recorded at $15^{\circ} \mathrm{C} 1$ hour after transfer into $\mathrm{D}_{2} \mathrm{O}$ buffer revealed difference features almost identical to those found with the 2 B27-TIS complexes (Figures $4 A$ and $B$ ), and these also resembled the corresponding IR spectral features of the pVIPRcomplexed $B^{\star} 2709$ and $B^{\star} 2705$ subtypes (Figure 4C). Moreover, the subtype-dependent spectral differences were much more pronounced than the spectral differences between 2 independent preparations of the corresponding HLA-B27 complexes. This demonstrates the high quality of the experimental data, as shown previously for complexes with the pVIPR peptide (24).

Comparison of IR spectroscopic data with results from x-ray crystallographic analyses. It is known that spontaneous structural fluctuations as well as local and global unfolding events can influence the exchange of amide protons with solvent deuterons $(27,28)$. Therefore, we next evaluated the crystallographic temperature factors (B factors) of the structures (Table 1), which highlight regions of different flexibility in the crystalline state $(11,12,15)$. 
An earlier assessment of the $B$ factors for the different structural units of $B^{*} 2709-p V I P R$ and $B^{*} 2705-$ pVIPR had failed to provide a clear-cut explanation for the observed subtle differences in amide proton protection between the 2 heavy chains (24). In the case of the pVIPR peptide, this might have been due to the different resolutions at which the 2 structures had been solved (Table 1). This explanation, however, cannot account for the differences between the HLA-B27 subtypes in complex with the 2 other peptides observed in the present study. For TIS, very similar average $B$ factors of $31.1 \AA 2$ $\left(B^{*} 2709\right)$ and $34.7 \AA 2\left(B^{*} 2705\right)$ were found, while clearly distinct values of $10.3 \AA 2$ and $19.6 \AA 2$, respectively, were observed in case of pLMP2, affecting residues at positions 7 and 8 , in particular (12) (Table 1). The B factors for pLMP2 thus revealed that this peptide is more flexibly bound in $B^{\star} 2705$ than in $B^{*} 2709$, despite the salt bridge-mediated anchoring of its middle through the pArg-5Asp-116 interaction (Figure 1).

\section{Discussion}

Water molecules within and around proteins are of overwhelming importance in stabilizing their tertiary and quarternary structures, and in so doing, are directly responsible for protein function (31). For example, such molecules are involved in the structural and functional activation of $\mathrm{G}$ protein-coupled receptors such as rhodopsin (32), and the water shell around the myoglobin molecule powers and controls internal motions such as ligand migration (31). An analysis of conserved water molecules in major histocompatibility complex (MHC) class I complexes revealed the presence of 3 water clusters in nearly all of the structures (33). Distinct locations of solvent molecules within the binding groove of different HLA-B27 subtypes, even when the same peptide is presented, must obviously be initiated by water molecules that surround the polymorphic heavy chain residue 116 within the $F$ pocket (for detailed images of such water networks, see refs. 14 and 29). Similarly, a polymorphic residue within the binding groove influences the shape of water networks in HLA-B44 subtypes (34).

We have previously reasoned that differential peptide flexibility in HLA-B27 subtypes presenting pLMP2 is most likely the consequence of a network of solvent molecules within the peptide-binding groove that is tighter in $B^{*} 2709$ than in $B^{*} 2705$, where the hydrophobic section of the pArg- 5 side chain prevents its formation (12) (Figure 1). The water network in the $\mathrm{F}$ pocket was also found to be denser in the less flexible $B^{*} 2709-$ TIS complex than in the $B^{*} 2705-$ TIS structure(15), while the dual conformation of the pVIPR peptide in the $B^{*} 2705$ subtype precludes direct comparison with $B^{*} 2709$ (11) (Figure 1). In contrast, the solvent molecule cluster is tighter in the complex of $B^{\star} 2705$ with the model peptide $\mathrm{m} 9$ (GRFAAAIAK) than in $\mathrm{B}^{\star} 2709-\mathrm{m} 9$, although an additional water molecule is part of the cluster that is found in $B^{\star} 2705$ (29). This complex is strikingly more stable than the $B^{\star} 2709-m 9$ complex $(22,35)$ and exhibits reduced binding groove flexibility $(21,36)$.

Although peptides with a C-terminal lysine, such as $\mathrm{m} 9$, cannot bind to $\mathrm{B}^{\star} 2709$ under natural conditions $(9,10)$, studies with the pLMP2, TIS, and m9 peptides allow one to correlate elevated heavy chain flexibility with the presence of a less favorably shaped water network within a peptide-binding groove and to link these characteristics to the Asp116His polymorphism. It seems unlikely, however, that the degree of differential heavy chain flexibility can be appreciably influenced by the lack (bacterially expressed heavy chain) or the presence of the glycosyl moiety on the heavy chain, since glycosylation occurs at the nonpolymorphic position Asn-86 (37), at a considerable distance from the flexible end of the a1-helix (see below).

IR spectroscopy cannot be used to localize the regions where the 2 heavy chains differ, but molecular dynamics simulations of complexes of HLA-B27 subtypes with pVIPR have shown increased flexibility of the $B^{*} 2705$ binding groove in comparison with that of $B^{*} 2709$ (24), thus corroborating the global isotopeedited IR spectroscopic findings. Specifically, the canonical conformation of pVIPR in the $B^{*} 2705$ subtype (Figure 1) induces increased flexibility of both $\alpha$-helices of the heavy chain in the region of amino acid residues 75-80 (end of the a 1-helix) and 137-150 (beginning of the a 2-helix), i.e., in the vicinity of and "above" the polymorphic residue 116. These segments of the 2 a-helices are always connected by hydrogen bonds to a bound peptide (5): the polymorphic heavy chain residue 77 (Asp, in the case of $B^{*} 2705$ or $B^{*} 2709$ ) contacts the peptide C-terminal amino acid, while the conserved heavy chain residue Trp-147 binds the penultimate peptide residue (Figure 5).

It therefore seems plausible that the Asp116His exchange and the consequential repositioning of water molecules exert an influence, by way of the suspended peptide, on the flexibility of the 2 opposing helical segments of the binding groove. Since the peptide contacts heavy chain residues 77 
and 147 through main chain atoms, the flexibility of the 2 a-helical segments should be independent of the sequence of a peptide. This is precisely what the present comparative study with 3 distinct ligands demonstrates. The last 2 residues of the peptides used in this study are Arg-Leu (TIS peptide), Thr-Val (pLMP2 peptide), and His-Leu (pVIPR peptide) (Figure 1).

Hydrophobic amino acids such as Leu or Val are the preferred C-terminal anchors of B27-bound peptides, but basic amino acids (Arg, Lys) can also occur (10). However, the latter are either absent from peptides found in $B^{*} 2709$ (in the case of Lys) or are only very rarely observed in this subtype (in the case of Arg). It is not known whether the $B^{\star} 2705$ heavy chain would be similarly flexible in bound peptides that contain a $\mathrm{C}$-terminal basic residue, but molecular dynamics simulations of the $\mathrm{m} 9$ peptide (see above) suggest that this might not be so $(21,36)$. Therefore, firm anchoring of specific residues of a peptide through salt bridges at the C-terminus $(29,38)$ might lead to reduced flexibility of the $B^{\star} 2705$ heavy chain. On the other hand, the present results demonstrate that the additional anchoring of a peptide through a centrally located residue that contacts Asp-116 (12) (Figures 1 and 5B) does not appear to influence heavy chain flexibility. Thus, the observed differential dynamic behavior of the 2 HLA-B27 subtypes at physiologic temperature has to be regarded as an intrinsic attribute of the heavy chain of the 2 subtypes, and not as a peptide sequence- or conformationinduced characteristic.

Two questions come to mind when one tries to reconcile the findings presented here with known properties of HLA-B27 molecules: 1) How could dynamic features of histocompatibility antigens be influenced by a deeply buried binding groove polymorphism? and 2) Would these biophysical characteristics have to be taken into account when attempting to explain the molecular basis of AS pathogenesis?

As discussed above, exchanges at the polymorphic residue 116 are invariably accompanied by alterations in the network of water molecules within the peptide-binding groove $(6,11-15,20,29)$. In the few studies in which this has been investigated $(12,15,21,29,36)$, tighter packing has been found in the less flexible HLA-B27 subtype. Looser water molecule packing, on the other hand, would permit the peptide main chain to be more flexible and would also lead to increased dynamics of the 2 a-helices, since the last $2 \mathrm{C}$-terminal peptide residues are connected to the 2 a-helices through highly conserved contacts $(5,20)$ (Figure 5).

Heavy chain dynamics are thus dependent not only on the polymorphic residue 116 and the distinct water cluster that surrounds its side chain, but also on the presence of a peptide. However, heavy chain flexibility is at the same time independent from the sequence and conformation of the bound peptide (Figure 4), establishing heavy chain dynamics as a novel characteristic that distinguishes $B^{*} 2705$ and $B^{*} 2709$. Since the function of a protein is inescapably connected to its dynamics $(31,39)$, altered conformational plasticity of class I MHC complexes will influence their interaction with ligands.

Therefore, and particularly with regard to the relevance of these findings for AS pathogenesis, the most plausible assumption may be that the flexibility of the a-helices exerts an effect on the interaction with $T$ cell receptors (TCRs) and killer cell immunoglobulinlike receptors (KIRs) on effector cells $(40,41)$. Causal involvement of CD8+ cytotoxic T lymphocytes in spondylarthritides in B*2705/human $\mathrm{B}_{2} \mathrm{~m}$-transgenic rats has recently been excluded (42). Although the situation in humans could obviously be different, these results do suggest that models of AS pathogenesis in which CD8+T cells do not likely play a decisive role should be considered. Since KIR molecules on natural killer (NK) cells interact with those regions of HLA-C antigens that exhibit homology with flexible $\alpha$-helical segments on $B^{\star} 2705$-peptide complexes $(24,41)$, we will briefly consider the possible consequences of our findings with regard to NK cell reactivity.

HLA class I-KIR interfaces exhibit striking charge complementarity. The interaction is characterized by a high energy threshold for recognition (41) and

in many ways resembles the observed interaction between the CD2 and CD58 cell surface antigens (43). HLA and KIR molecules typically behave as rigid bodies, quite unlike the situation in HLA-TCR interactions in which, in particular, the TCR loops can be very flexible (40). Therefore, elevated dynamics of the HLA molecule, as seen in the $B^{\star} 2705$ subtype presenting peptides with aliphatic Ctermini, could result in impaired recognition of the complex by inhibitory KIRs (KIR3DL1 in the case of HLA-B27 molecules). In turn, this might lead to activation of NK cells, and possibly even lysis of the target cell. KIR molecules that recognize HLA-B27 antigens not only are numerous, but are also polymorphic, and their combined actions on cells expressing HLA-B27 molecules are insufficiently understood (ref. 44; for review, see ref. 45). Nevertheless, it is reasonable to assume that KIRs might play a role in AS pathogenesis, and our finding of differential flexibility between 2 HLA-B27 subtypes in a region of the binding groove that is recognized by these receptors offers the opportunity to relate 
their activity to a subtype-intrinsic, peptide sequence- and conformation-independent heavy chain property that is a consequence of micropolymorphism at heavy chain position 116 .

Finally, it should be kept in mind that dynamic characteristics of the binding groove also likely play a role in the process of loading the molecule with a suitable peptide within the endoplasmic reticulum (46-48). Notably, B*2705 and other AS-associated subtypes fold much more slowly than subtypes that are not associated with AS (e.g., $\mathrm{B}^{\star} 2709$ ) (46), and this is also observed when complexes with the heavy chain of various subtypes are reconstituted in vitro (Huser $\mathrm{H}$, Uchanska-Ziegler B: unpublished observations). It is not known whether these differences can be regarded as a consequence of distinct heavy chain flexibility, but in the absence of another explanation, this possibility should at least be considered.

In conclusion, although the findings of the present study do not explain the involvement of HLA-B27 antigens in AS pathogenesis, they provide a picture of these molecules that goes beyond the purely static view derived from conventional $x$-ray crystallographic analyses. Nuclear magnetic resonance spectroscopic analyses of HLA-B27 subtypes that are differentially associated with AS might eventually enable investigators to merge the structural and dynamic features of these subtypes and further elucidate the differences between $B^{\star} 2705$ and $B^{\star} 2709$ that we have described herein.

\section{Acknowledgments}

We thank Dr. R. Misselwitz (Institut für Immungenetik, Charité-Universitätsmedizin Berlin) for useful suggestions and Ms Christina Schnick for help with the preparation of the HLA-B27 complexes.

\section{Author Contributions}

All authors were involved in drafting the article or revising it critically for important intellectual content, and all authors approved the final version to be published. Dr. Ziegler had full access to all of the data in the study and takes responsibility for the integrity of the data and the accuracy of the data analysis.

Study conception and design. Fabian, Ziegler, Naumann, Uchanska- Ziegler.

Acquisition of data. Fabian, Huser.

Analysis and interpretation of data. Fabian, Loll, Ziegler, Naumann, Uchanska-Ziegler. 


\section{References}

1. Lopez de Castro JA. HLA-B27 and the pathogenesis of spondyloarthropathies. Immunol Lett 2007;108:27-33.

2. Taurog JD. The mystery of HLA-B27: if it isn't one thing, it's another [editorial]. Arthritis Rheum 2007;56:2478-81.

3. Khan MA, Mathieu A, Sorrentino R, Akkoc N. The pathogenetic role of HLA-B27 and its subtypes. Autoimmun Rev 2007;6:183-9.

4. D'Amato M, Fiorillo MT, Carcassi C, Mathieu A, Zuccarelli PP, Bitti R, et al. Relevance of residue 116 of HLA-B27 in determining susceptibility to ankylosing spondylitis. Eur J Immunol 1995; 25:3199201.

5. Madden DR. The three-dimensional structure of peptide-MHC complexes. Annu Rev Immunol 1995;13:587-622.

6. Ziegler A, Loll B, Misselwitz R, Uchanska-Ziegler B. Implications of structural and thermodynamic studies of HLA-B27 subtypes exhibiting differential association with ankylosing spondylitis. Adv Exp Med Biol 2009;649:177-95.

7. Fiorillo MT, Cauli A, Carcassi C, Bitti PP, Vacca A, Passiu G, et al. Two distinctive HLA haplotypes harbor the B27 alleles negatively or positively associated with ankylosing spondylitis in Sardinia: implications for disease pathogenesis. Arthritis Rheum 2003; 48:1385-9.

8. Fiorillo MT, Maragno M, Butler R, Dupuis ML, Sorrentino R. CD8+ T-cell autoreactivity to an HLAB27-restricted self-epitope correlates with ankylosing spondylitis. J Clin Invest 2000;106: 47-53.

9. Ramos M, Paradela A, Vazquez M, Marina A, Vazquez J, Lopez de Castro JA. Differential association of HLA-B*2705 and $B^{*} 2709$ to ankylosing spondylitis correlates with limited peptide subsets but not with altered cell surface stability. J Biol Chem 2002;277:28749-56.

10. Lopez de Castro JA, Alvarez I, Marcilla M, Paradela M, Ramos M, Sesma L, et al. HLA-B27: a registry of constitutive peptide ligands. Tissue Antigens 2004;63:424-45.

11. Hulsmeyer M, Fiorillo MT, Bettosini F, Sorrentino R, Saenger W, Ziegler A, et al. Dual, HLA-B27 subtype-dependent conformation of a self-peptide. J Exp Med 2004;199:271-81.

12. Fiorillo MT, Ruckert C, Hulsmeyer M, Sorrentino R, Saenger W, Ziegler A, et al. Allele-dependent similarity between viral and self-peptide presentation by HLA-B27 subtypes. J Biol Chem 2005;280:2962-71.

13. Ruckert C, Fiorillo MT, Loll B, Moretti R, Biesiadka J, Saenger W, et al. Conformational dimorphism of self-peptides and molecular mimicry in a disease-associated HLA-B27 subtype. J Biol Chem 2006;281:2306-16.

14. Beltrami A, Rossmann M, Fiorillo MT, Paladini F, Sorrentino R, Saenger W, et al. Citrullinationdependent differential presentation of a self-peptide by HLA-B27 subtypes. J Biol Chem 2008; 283:27189-99.

15. Hulsmeyer M, Welfle K, Pohlmann T, Misselwitz R, Alexiev U, Welfle W, et al. Thermodynamic and structural equivalence of two HLA-B27 subtypes complexed with a self-peptide. J Mol Biol 2005;346:1367-79.

16. ColbertRA.HLA-B27misfolding: a solution to the spondyloarthropathy conundrum? Mol Med Today 2000;6:224-30.

17. Allen RL, O'Callaghan CA, McMichael AJ, Bowness P. Cutting edge: HLA-B27 can form a novel $B_{2}$-microglobulin-free heavy chain homodimer structure. J Immunol 1999;162:5045-8.

18. Hammer RE, Maika SD, Richardson JA, Tang JP, Taurog JD. Spontaneous inflammatory disease in transgenic rats expressing HLA-B27 and human $\beta_{2} \mathrm{~m}$ : an animal model of HLA-B27- associated human disorders. Cell 1990;63:1099-112.

19. Ramos M, Alvarez I, Sesma L, Logean A, Rognan D, Lopez de Castro JA. Molecular mimicry of an HLA-B27-derived ligand of arthritis-linked subtypes with chlamydial proteins. J Biol Chem 2002;277:37573-81.

20. Kumar P, Vahedi-Faridi A, Saenger W, Merino E, Lopez de Castro JA, Uchanska-Ziegler B, et al. Structural basis for T cell alloreactivity among three HLA-B14 and HLA-B27 antigens. J Biol Chem 2009;284:29784-97.

21. Pohlmann T, Bockmann RA, Grubmuller H, Uchanska-Ziegler B, Ziegler A, Alexiev U. Differential peptide dynamics is linked to MHC polymorphism. J Biol Chem 2004;279:28197-201.

22. Winkler K, Winter A, Ruckert C, Uchanska-Ziegler B, Alexiev U. Natural MHC class I polymorphism controls the pathway of peptide dissociation from HLA-B27 complexes. Biophys J 2007; 93:2743-55.

23. Narzi D, Winkler K, Saidowsky J, Misselwitz R, Ziegler A, Bockmann RA, et al. Molecular determinants of major histocompatibility complex class I complex stability: shaping antigenic features through short range and long range electrostatic interactions. J Biol Chem 2008;283:23093-103. 
24. Fabian H, Huser H, Narzi D, Misselwitz R, Loll B, Ziegler A, et al. HLA-B27 subtypes differentially associated with disease exhibit conformational differences in solution. J Mol Biol 2008;376: 798-810.

25. Taurog JD. Animal models of spondyloarthritis. Adv Exp Med Biol 2009;649:245-54.

26. Fabian H, Mantele W. Infrared spectroscopy of proteins. In: Chalmers JM, Griffiths PR, editors. Handbook of vibrational spectroscopy. Chichester (UK): John Wiley \& Sons; 2002. p. 3399-425.

27. Barth A. Infrared spectroscopy of proteins. Biochim Biophys Acta 2007;1767:1073-101.

28. Englander SW, Kallenbach NR. Hydrogen exchange and structural dynamics of proteins and nucleic acids [review]. Q Rev Biophys 1983;4:521-655.

29. Hulsmeyer M, Hillig RC, Volz A, Ruhl M, Schroder W, Saenger W, et al. HLA-B27 subtypes differentially associated with disease exhibit subtle structural alterations. J Biol Chem 2002;277: 47844-53.

30. Garboczy DN, Hung DT, Wiley DC. HLA-A2-peptide complexes: refolding and crystallization of molecules expressed in Escherichia coli and complexed with single antigenic peptides. Proc Natl Acad Sci U S A 1992;89:3429-33.

31. Frauenfelder $\mathrm{H}$, Chen G, Berendzen J, Fenimore PW, Jansson H, McMahon BH, et al. A unified model of protein dynamics. Proc Natl Acad Sci U S A 2009;106:5129-34.

32. Angel TE, Gupta S, Jastrzebska B, Palczewski K, Chance MR. Structural waters define a functional channel mediating activation of the GPCR, rhodopsin. Proc Natl Acad Sci U S A 2009;106:

14367-72.

33. Ogata K, Wodak SJ. Conserved water molecules in MHC class-I molecules and their putative structural and functional roles. Protein Eng 2002;15:697-705.

34. Macdonald WA, Purcell AW, Mifsud NA, Ely LK, Williams DS, Chang L, et al. A naturally selected dimorphism within the HLA-B44 supertype alters class I structure, peptide repertoire, and T cell recognition. J Exp Med 2003;198:679-91.

35. Hillig RC, Hulsmeyer M, Welfle K, Misselwitz R, Welfle W, Saenger W, et al. Thermodynamic and structural analysis of peptide- and allele-dependent properties of two HLA-B27 subtypes exhibiting differential disease association. J Biol Chem 2004;279:652-63.

36. Ziegler A, Muller CA, Bockmann RA, Uchanska-Ziegler B. Low-affinity peptides and T-cell selection. Trends Immunol 2009; 30:53-61.

37. Bjorkman PJ, Parham P. Structure, function, and diversity of class I major histocompatibility complex molecules. Annu Rev Biochem 1990;59:253-88.

38. Stewart-Jones GB, di Gleria K, Kollnberger S, McMichael AJ, Jones EY, Bowness P. Crystal structures and KIR3DL1 recognition of three immunodominant viral peptides complexed to HLA$B^{*} 2705$. Eur J Immunol 2005;35:341-51.

39. Henzler-Wildman K, Kern D. Dynamic personalities of proteins. Nature 2007;450:964-72.

40. Rudolph MG, Stanfield RL, Wilson IA. How TCRs bind MHCs, peptides, and coreceptors. Annu Rev Immunol 2006;24:419-66.

41. Boyington JC, Sun PD. A structural perspective on MHC class I recognition by killer cell immunoglobulin-like receptors. Mol Immunol 2002;38:1007-21.

42. Taurog JD, Dorris ML, Satumtira N, Tran TM, Sharma R, Dressel R, et al. Spondylarthritis in HLAB27/human $B_{2}$-microglobulin- transgenic rats is not prevented by lack of CD8. Arthritis Rheum 2009;60:1977-84.

43. Wang JH, Smolyar A, Tan K, Liu JH, Kim M, Sun ZY, et al. Structure of a heterophilic adhesion complex between the human CD2 and CD58 (LFA-3) counterreceptors. Cell 1999;97:791-803.

44. Kollnberger S, Chan A, Sun MY, Chen LY, Wright C, di Gleria K, et al. Interaction of HLA-B27 homodimers with KIR3DL1 and KIR3DL2, unlike HLA-B27 heterotrimers, is independent of the sequence of bound peptide. Eur J Immunol 2007;37:1313-22.

45. Diaz-Pena R, Blanco-Gelaz MA, Lopez-Larrea C. KIR genes and their role in spondyloarthropathies. Adv Exp Med Biol 2009;649: 286-99.

46. Galocha B, Lopez de Castro JA. Folding of HLA-B27 subtypes is determined by the global effect of polymorphic residues and shows incomplete correspondence to ankylosing spondylitis. Arthritis Rheum 2008;58:401-12.

47. Blanco-Gelaz MA, Suarez-Alvarez B, Diaz-Pena R, Lopez-Larrea C. HLA-B27 polymorphism at position 116 critically influences the association with TAP/tapasin, intracellular trafficking and conformational homodimers formation. Mol Immunol 2009;46: 1304-11.

48. Dong G, Wearsch PA, Peaper DR, Cresswell P, Reinisch KM. Insights into MHC class I peptide loading from the structure of the tapasin-ERp57 thiol oxioreductase heterodimer. Immunity 2009; $30: 21-32$. 
Tables and Figures

Table 1. Structural characteristics of the HLA-B27-peptide complexes Analyzed

\begin{tabular}{lccr}
\hline \multicolumn{1}{c}{ Complex } & $\begin{array}{c}\text { Resolution } \\
(\AA)\end{array}$ & $\begin{array}{c}\text { Average B factor } \\
\left(\AA^{2}\right)^{*}\end{array}$ & $\begin{array}{c}\text { PDB entry } \\
(\text { ref. }) \dagger\end{array}$ \\
\hline B*2705-TIS & 2.27 & 34.7 & 1WOV (15) \\
B*2709-TIS & 2.10 & 31.1 & 1WOW (15) \\
B*2705-pLMP2 & 1.55 & 19.6 & 1UXS (12) \\
B*2709-pLMP2 $^{*}$ & 1.72 & 10.3 & 1UXW (12) \\
B*2705-pVIPR & 1.47 & 16.5 & 1OGT (11) \\
B*2709-pVIPR $^{*}$ & 2.20 & 35.2 & 1OF2 (11) \\
\hline
\end{tabular}

* Average temperature factor (B factor) for all peptide residues.

$\dagger$ References cited are those in which the crystal structure of the given B27-peptide complex has been published. PDB = Protein Data Bank.

\section{Figure 1}
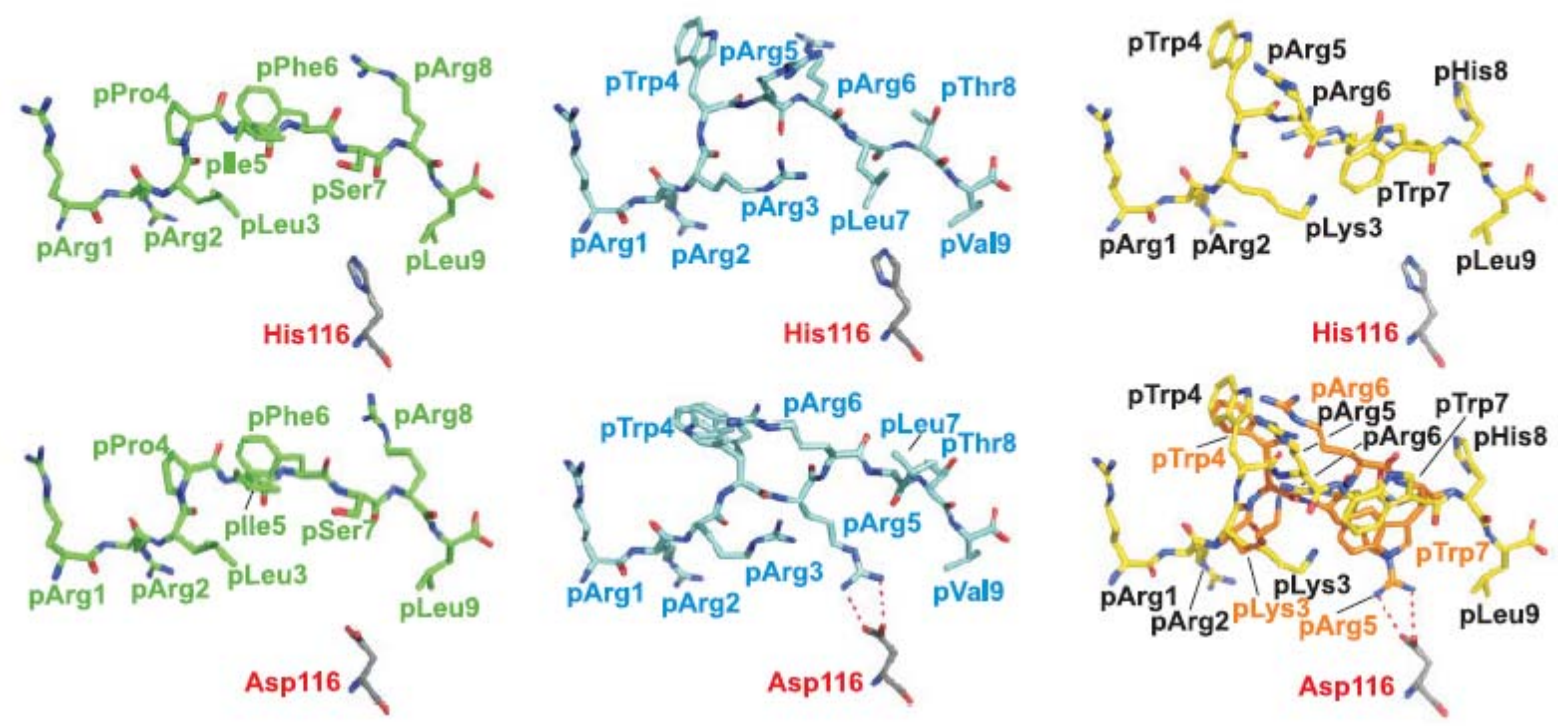

Conformations of the peptides TIS (left), pLMP2 (middle), and pVIPR (right) within the peptide-binding groove of the $B^{*} 2709$ and $B^{*} 2705$ subtypes. The peptide conformations are shown in complex with $\mathrm{B}^{\star} 2709$ (top) and $\mathrm{B}^{\star} 2705$ (bottom). Only the TIS peptide is displayed very similarly by both subtypes, while the middle part of pLMP2 is bound in drastically different conformations due to the formation of a salt bridge between pArg-5 of this peptide and Asp-116 of the heavy chain, which is possible only in $\mathrm{B}^{*} 2705$. In addition, while TIS (15) and pLMP2 (12) are bound by both B27 subtypes in a single conformation, for pVIPR this is observed only in the case of $B^{*} 2709$ (11). In contrast, the binding of this peptide by $B^{*} 2705$ occurs in a unique dual conformation with roughly equal occupancy. While one of the binding modes resembles that found in $B^{*} 2709$ (yellow), the other (orange) is distinct from the first between pLys-3 and pTrp-7. It is characterized by the formation of a salt bridge between pArg-5 and Asp-116 and is thus similar to the single conformation of pLMP2 when bound to B*2705 (12). 

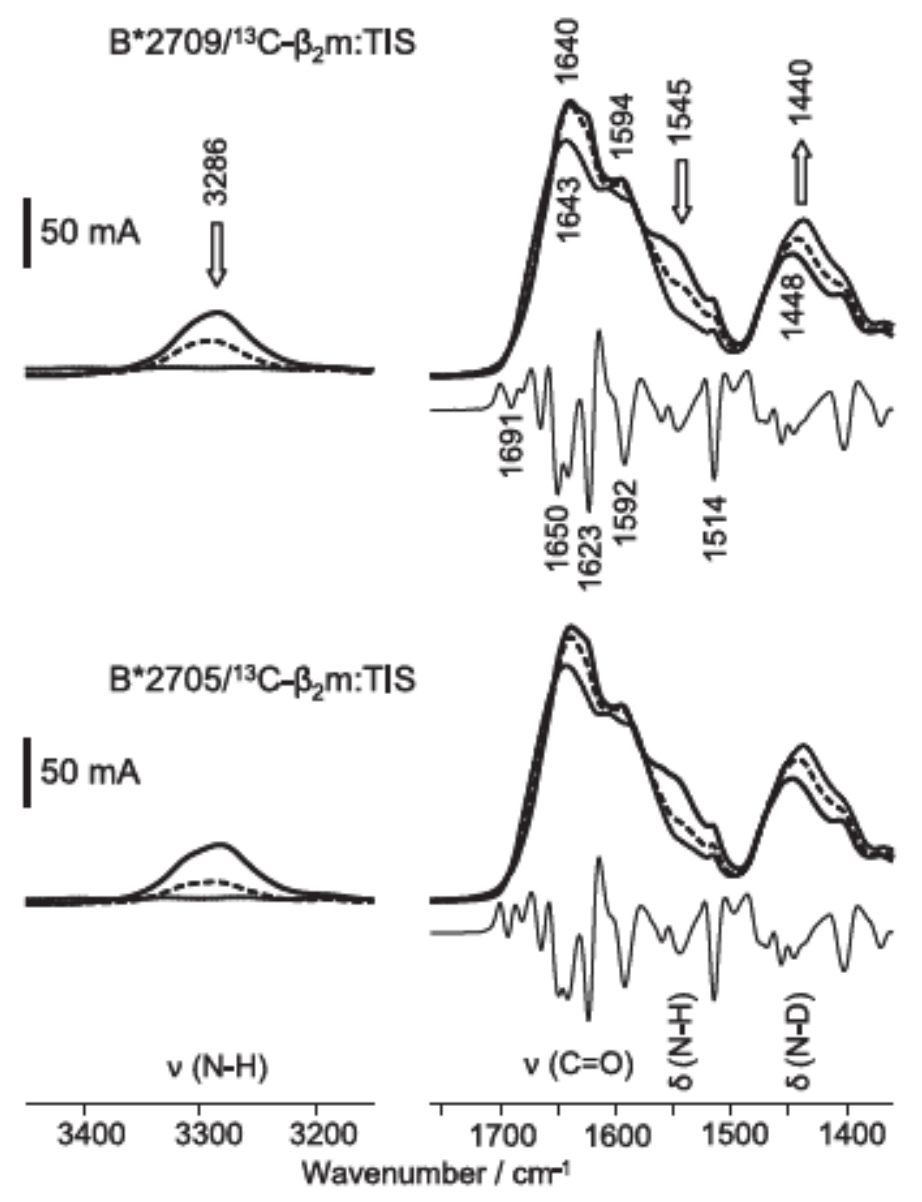

Infrared (IR) absorbance spectra $B^{\star} 2709$ and $B^{*} 2705$, each complexed with ${ }^{13} \mathrm{C}-\mathrm{B}_{2}$-microglobulin and TIS. $B^{*} 2705,{ }^{13} \mathrm{C}-\beta_{2} \mathrm{~m}$, and TIS. $B^{*} 2709-{ }^{13} \mathrm{C}-\beta_{2} \mathrm{~m}-\mathrm{TIS}$ and $\mathrm{B}^{*} 2705-{ }^{13} \mathrm{C}-\beta_{2} \mathrm{~m}-\mathrm{TIS}$ were measured at $15^{\circ} \mathrm{C}$ (thick lines), $60^{\circ} \mathrm{C}$ (dashed lines), and $90^{\circ} \mathrm{C}$ (dotted lines), 1 hour after transfer into $\mathrm{D}_{2} \mathrm{O}$ buffer. The IR spectra in the amide I region $(\mathrm{C}=\mathrm{O}$ stretching vibration of the peptide bond) are characterized by a maximum corresponding to the heavy chain $\left(1,640 \mathrm{~cm}^{-1}\right)$ and a pronounced shoulder at $\sim 1,594$ $\mathrm{cm}^{-1}$, which indicates the presence of $\beta$-sheet structure of ${ }^{13} \mathrm{C}$-labeled $\beta_{2} \mathrm{~m}$ in the complex. The weak feature at $\sim 1,545 \mathrm{~cm}^{-1}$ is due primarily to the amide II band ( $\mathrm{N}-\mathrm{H}$ bending vibration strongly coupled to $\mathrm{C}-\mathrm{N}$ stretching) of nonexchanged amide backbone groups of the heavy chain. Exchange of protons with deuterons is accompanied by a decrease of the band intensity at $\sim 1,545 \mathrm{~cm}^{-1}$ and an increase of band intensity at $\sim 1,445 \mathrm{~cm}^{-1}$ (amide II'). Residual nonexchanged $\mathrm{N}-\mathrm{H}$ groups are directly indicated by a positive band at $\sim 3,286 \mathrm{~cm}^{-1}$ (amide A). The thin traces represent the second derivatives of the spectra for each of the complexes at $15^{\circ} \mathrm{C}$. The 6 negative peaks between $1,620 \mathrm{~cm}^{-1}$ and $1,700 \mathrm{~cm}^{-1}$ are due to amide I band components of secondary structure elements of the heavy chain. 
Figure 3

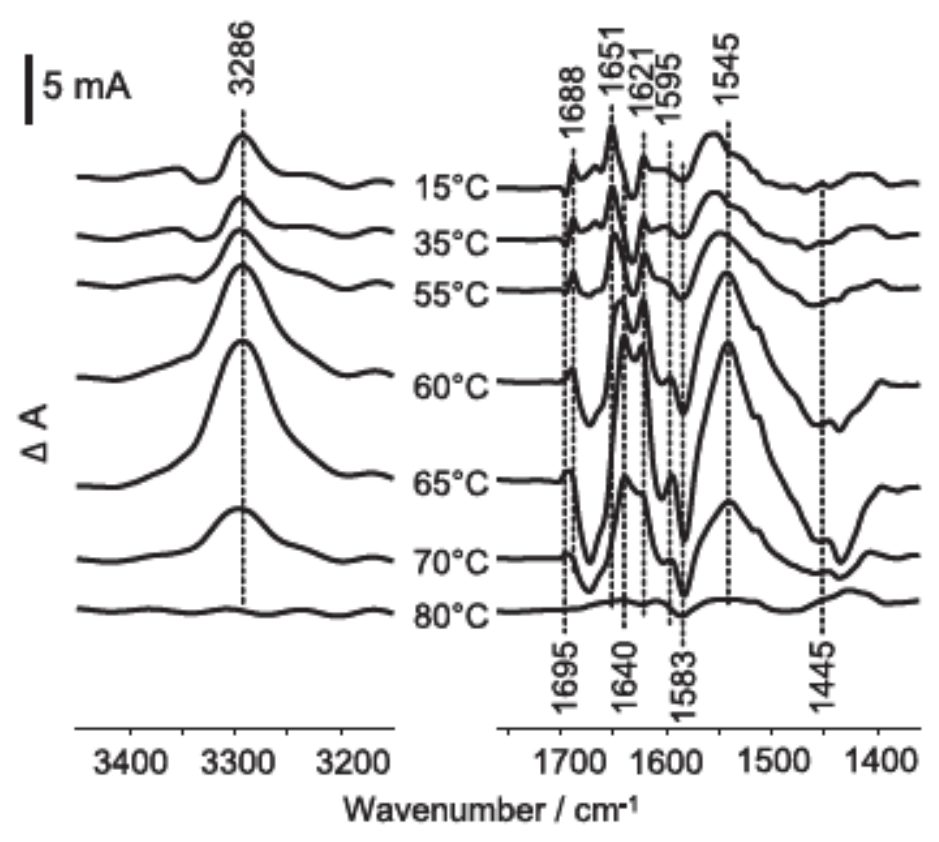

IR difference spectra between $B^{*} 2709-{ }^{13} C-B_{2} m-T I S$ and $B^{*} 2705-{ }^{13} C-B_{2} m-T I S$. Difference spectra were obtained by subtracting the absorbance spectrum of $B^{*} 2705-{ }^{13} C-B_{2} m-T I S$ from that of $B^{*} 2709$ ${ }^{13} \mathrm{C}-\beta_{2} \mathrm{~m}-\mathrm{TIS}$ at the indicated temperatures. The first spectrum of each sample (at $15^{\circ} \mathrm{C}$ ) was obtained 1 hour after transfer into $D_{2} \mathrm{O}$ buffer. The spectra of the 2 samples were normalized using the intensity of the tyrosine band at $1,514 \mathrm{~cm}^{-1}$ as an internal standard. The component at $\sim 1,550 \mathrm{~cm}^{-1}$ and a corresponding negative feature at $\sim 1,445 \mathrm{~cm}^{-1}$, together with a positive band at $\sim 3,286 \mathrm{~cm}^{-1}$, indicate a slightly lower degree of hydrogen/deuterium exchange of the amide groups of the $B^{*} 2709$ heavy chain compared with the $B^{\star} 2705$ heavy chain at low temperatures. Note that the ordinate of the difference spectra has been expanded by a factor of 10 compared with the ordinate of the absorbance spectra shown in Figure 2. See Figure 2 for explanations and definitions. 


\section{Figure 4}

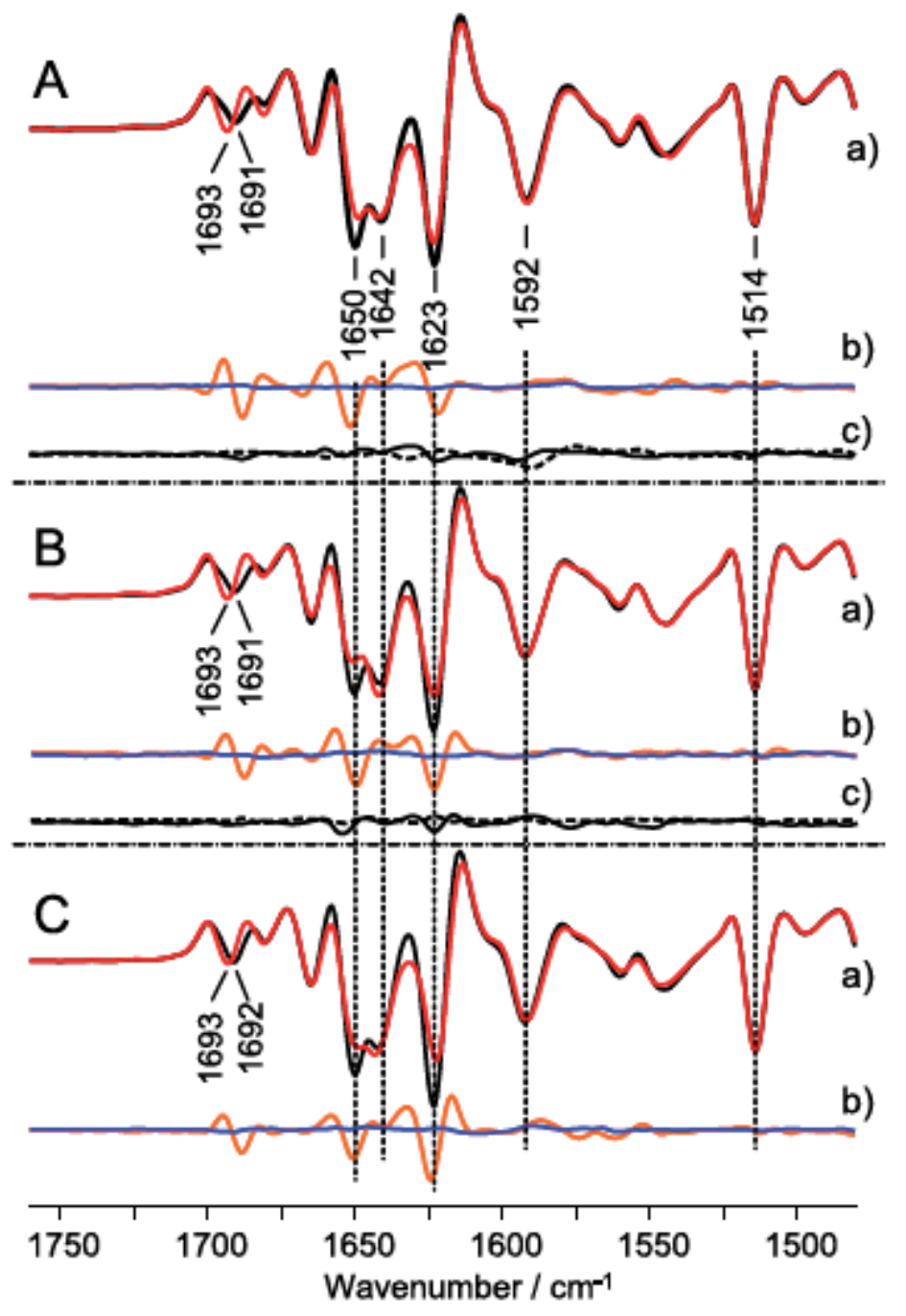

Peptide-independence of increased heavy chain flexibility of $B^{*} 2705$ complexes. The second derivative IR spectra of the TIScomplexed (A), pLMP2-complexed (B), and pVIPR-complexed (C) $B^{*} 2709-{ }^{13} \mathrm{C}-\beta_{2} \mathrm{~m}$ and $\mathrm{B}^{*} 2705-{ }^{13} \mathrm{C}-\beta_{2} \mathrm{~m}$ (black and red lines in part $\mathbf{a}$ in each panel, respectively), at a temperature of $15^{\circ} \mathrm{C}, 1$ hour after transfer into $\mathrm{D}_{2} \mathrm{O}$ buffer, are shown. (Data in $\mathbf{C}$ are from previous work by our group [24].) Part $\mathbf{b}$ in each panel depicts the IR difference spectra ( $B^{\star} 2709$ minus $\left.B^{\star} 2705\right)$ of the second derivatives at $15^{\circ} \mathrm{C}$ (orange traces) and at $90^{\circ} \mathrm{C}$ (blue traces). The low-temperature IR difference spectra provide evidence of a very close similarity of the structure and flexibility between the 2 complexes with the 3 peptides. The high-temperature IR difference spectra are flat, demonstrating the loss of all differences between the HLA-B27 subtypes that were apparent at low temperatures. Part $\mathbf{C}$ in $\mathbf{A}$ and $\mathbf{B}$ depicts the IR difference spectra of the second derivatives of experiments with 2 independent preparations of each HLA-B27-peptide complex (continuous traces represent HLA$B^{\star} 2709$; dashed traces represent $\mathrm{HLA}-\mathrm{B}^{\star} 2705$ ) at $15^{\circ} \mathrm{C}$, demonstrating the high reproducibility of the experimental data. See Figure 2 for explanations and definitions. 
Figure 5
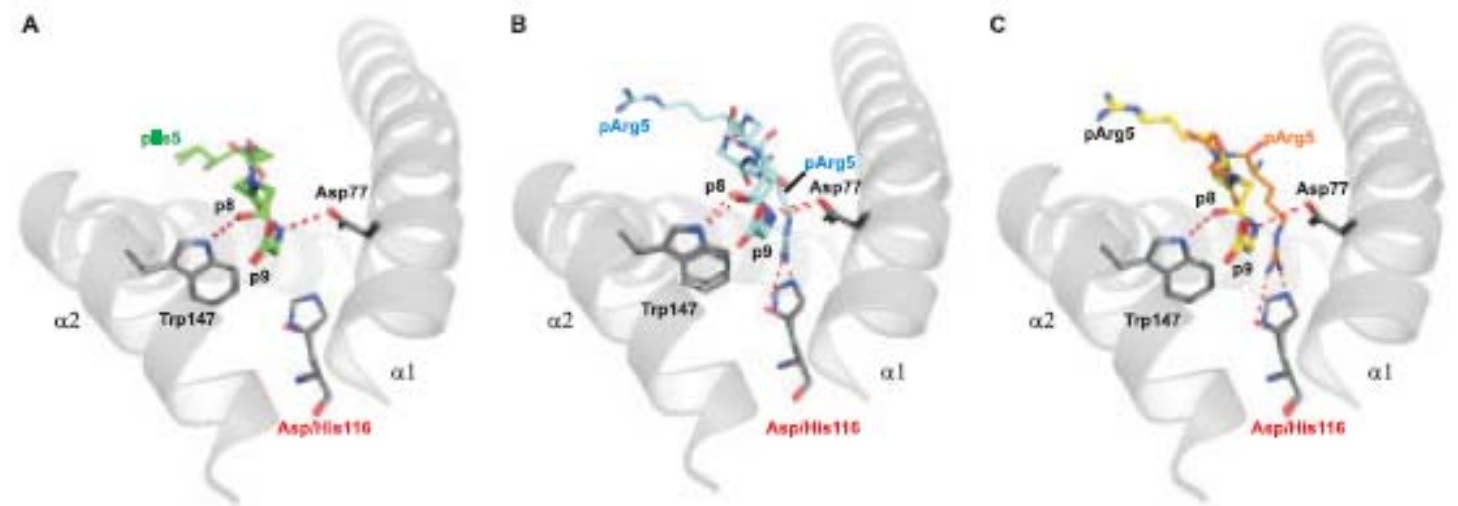

Conserved interactions between a displayed peptide and a-helices of the peptide-binding groove. The interactions of the peptide residues p8 (main chain carbonyl) and Trp-147 (side chain NE atom) and of p9 (main chain amide) and Asp-77 (side chain OD1 atom) in the TIS (A), pLMP2 (B), and pVIPR (C) peptides are depicted. The structures of $B^{\star} 2709$ and $B^{\star} 2705$ are superimposed; oxygen atoms are shown in red and nitrogen atoms in blue. The view is from a slightly "elevated" position near the carboxy-terminal amino acid, roughly parallel to the peptide main chain. Only the peptide segments from p5 to p9 are shown, and side chains are omitted with the exception of plle-5 (TIS) and pArg-5 (pLMP2 and pVIPR). The latter residue points in different directions in the 2 subtypes (shown in the same color [blue] for pLMP2) or within the $\mathrm{B}^{*} 2705$ subtype (noncanonical peptide conformation shown in orange for pVIPR). Hydrogen bonds (peptide- $\alpha$-helix interactions) and salt bridges (pArg-5-Asp116 interactions) are depicted as dotted lines. Differences in peptide sequences and conformations still permit the formation of highly conserved hydrogen bonds between the $\alpha$-helices ( $\alpha 1$ and $\alpha 2$ ) and peptide main chain atoms in all 6 structures. 